\title{
A new tribological experimental setup to study confined and sheared monolayers
}

Cite as: Rev. Sci. Instrum. 87, 033903 (2016); https://doi.org/10.1063/1.4943670

Submitted: 19 August 2015 . Accepted: 28 February 2016 . Published Online: 18 March 2016

L. Fu (D), D. Favier, T. Charitat, C. Gauthier, and A. Rubin (D)

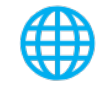

\section{ARTICLES YOU MAY BE INTERESTED IN}

A micro-nano-rheometer for the mechanics of soft matter at interfaces

Review of Scientific Instruments 87, 113906 (2016); https://doi.org/10.1063/1.4967713

Forced oscillations dynamic tribometer with real-time insights of lubricated interfaces Review of Scientific Instruments 88, 035101 (2017); https://doi.org/10.1063/1.4977234

Osmotic and diffusio-osmotic flow generation at high solute concentration. II. Molecular dynamics simulations

The Journal of Chemical Physics 146, 194702 (2017); https://doi.org/10.1063/1.4981794

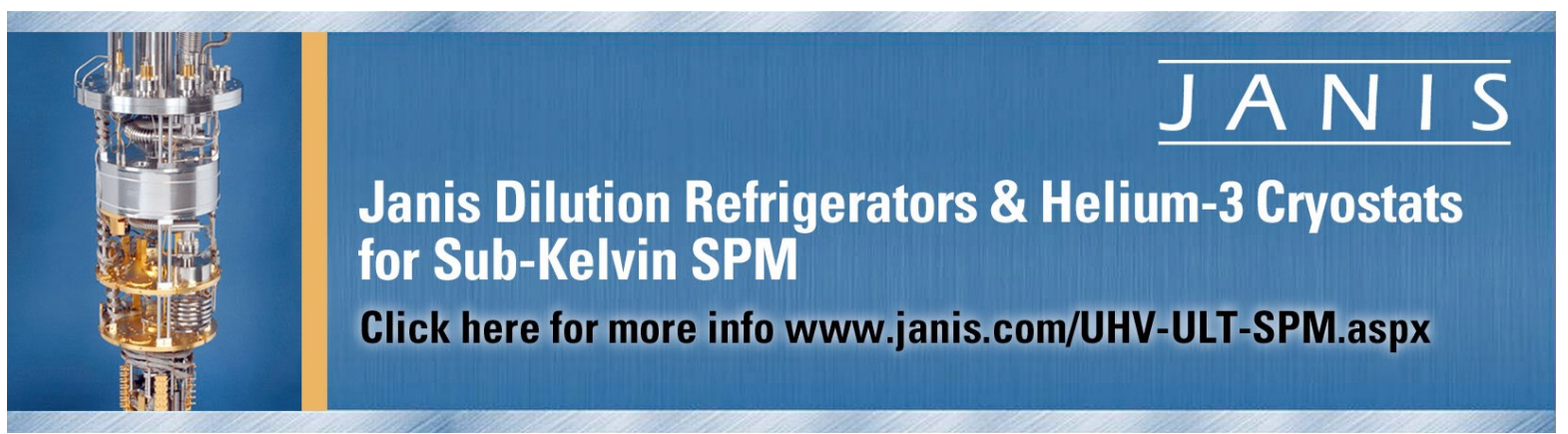




\title{
A new tribological experimental setup to study confined and sheared monolayers
}

\author{
L. Fu, D. Favier, T. Charitat, C. Gauthier, and A. Rubin \\ UPR22/CNRS, Institut Charles Sadron, Université de Strasbourg, 23 rue du Loess, BP 84047, \\ 67034 Strasbourg Cedex 2, France
}

(Received 19 August 2015; accepted 28 February 2016; published online 18 March 2016)

\begin{abstract}
We have developed an original experimental setup, coupling tribology, and velocimetry experiments together with a direct visualization of the contact. The significant interest of the setup is to measure simultaneously the apparent friction coefficient and the velocity of confined layers down to molecular scale. The major challenge of this experimental coupling is to catch information on a nanometer-thick sheared zone confined between a rigid spherical indenter of millimetric radius sliding on a flat surface at constant speed. In order to demonstrate the accuracy of this setup to investigate nanometer-scale sliding layers, we studied a model lipid monolayer deposited on glass slides. It shows that our experimental setup will, therefore, help to highlight the hydrodynamic of such sheared confined layers in lubrication, biolubrication, or friction on solid polymer. (C) 2016 AIP Publishing LLC. [http://dx.doi.org/10.1063/1.4943670]
\end{abstract}

\section{INTRODUCTION}

Many industrial contact applications lead to high confinement conditions between two sliding surfaces (MPa or GPa range) where the use of lubricating films significantly reduces friction. The frictional process is governed by different lubrication mechanisms depending on the thickness and viscosity of the lubricating film as described by the Stribeck curve that distinguishes boundary, mixed, and elastohydrodynamic lubrication regimes. ${ }^{1-4}$ From the 1980 s, mixed and boundary regimes were widely investigated as huge advances arose in instrumentation dedicated to atomic or molecular scales. Two fields have evolved in parallel: (i) nanotribology, dealing with interfacial problems of highly confined sheared molecularly thin films and mainly studied by two major techniques: surface-force apparatus (SFA) and atomic-force microscopes $(\mathrm{AFM})^{5}$ and (ii) more recently, the development of microfluidic devices leading to a renewed interest on the nature of interfacial slip velocity of sheared fluid-solid interfaces. It was mainly studied by laser velocimetry techniques ${ }^{6}$ or dynamic surface force apparatus. ${ }^{7-9}$ These two fields give valuable information for complex materials which both have fluid and solid behavior under confinement and shear.

In surface forces studies of highly confined thin films, the key parameters are mainly the molecular roughness of the surfaces, the molecular nature of involved fluids, and the surface-fluid interaction. ${ }^{10}$ SFA devices were widely employed to study hydrodynamics and flow boundary conditions of confined Newtonian liquids ${ }^{7,11,12}$ and confined biological membranes. ${ }^{8,9}$ In both cases, SFA experimental investigations are resolved at nanometer scales and showed that highly confined films' behaviours deviate from bulk properties. ${ }^{13}$ Information on shearing can be obtained indirectly by a normal approach of the counter mica surface at constant speed ${ }^{14}$ or with small amplitude oscillations normal to the plane. ${ }^{7,9,11}$ For Newtonian liquids, transitions were probed and described by the stick-slip model proposed by Israelachvili, ${ }^{13}$ where, in sheared thin films, flow motion starts from finite yield points. At the solid-liquid interface, a so-called immobile layer was probed and estimated to a few molecular layers (1-5). For biological membranes, the slip plane was located at the weakest molecular interaction and depends on hydrophilicity of used molecules. The molecular friction of the gel-phased supported bilayers confined under water was deduced from these measurements: ${ }^{9}$ dynamic mode SFA was used as a nanorheological device. Dynamic mode SFA allows therefore to study elastic, viscoelastic properties, and effective viscosity of confined molecularly thin layers. Small amplitude lateral perturbations were also introduced using a simple piezoelectric tube ${ }^{15}$ or a couple of piezoelectric bimorphs. ${ }^{16}$ The main difference lies in the used model to extract the contribution of the confined thin layer from the mechanical compliance of the device. In this shear mode configuration, mica counter surfaces can move parallel to each other at a constant surface separation like in a classical rheometer. Liquid-to-solid phase transitions of confined molecularly thin layers from measured stick-slip motion depending on the state of the fluid (more liquid-like or solid-like) were probed. An alternative experimental method was performed at resonance conditions to characterize elastic parameters of confined thin film. ${ }^{17}$ Switching from Newtonian liquids to confined LangmuirBlodgett (LB) layers, similar experiments were performed. For instance, the group of Kutzner et al. confirmed the previous work of Briscoe on confined LB layers: ${ }^{18}$ hydrocarbon chain length of phospholipid monolayers has little influence on shear force. Elastic modulus and viscosity of the phospholipid monolayers decrease with contact stress below $1 \mathrm{MPa}^{19}$ and up to $10 \mathrm{MPa}^{20}$ Contact pressure seems to decrease relaxation times. These studies brought major understanding on molecular shearing mechanisms. But the main limitation of these modified SFAs is that small oscillatory shear and contact stresses rarely above $10 \mathrm{MPa}$ are not sufficient to understand shear response of high confined lubricant. In AFM devices, interfacial forces measurements provide information about 
adhesion, fracture, and tribology. Gradually, improvements of tribology analysis were made: deflection force sensors were adapted $^{21}$ and Lateral Force Microscope (LFM) emerged. Some authors performed experiments with LFM and a macrotribometer to correlate nano- and macro-studies on metallic surfaces. ${ }^{22,23}$ This technique was then extended to friction on polymeric surfaces such as LB films. ${ }^{24}$ Gourdon et al. highlighted some anisotropy in molecular organization during friction, for high mean contact pressure. ${ }^{25}$ Usual interpretation of the measurements is done with the Hertz model, but the AFM/LFM tip geometry leads to contact pressure up to few GPa that is a plastic contact. From an experimental point of view, covering a wide range of sliding speed is not always easy. Tambe et al. covers 4 speed decades up to $10 \mathrm{~mm} \mathrm{~s}^{-1}$ on scan length of 2 and $25 \mu \mathrm{m}$ with a modified AFM setup. ${ }^{26}$ This study shows that for a self-assembled monolayer, the friction is linearly dependent on the velocity below $100 \mu \mathrm{m} \mathrm{s}^{-1}$ and constant above. At low velocity, these molecules reorient, but this study gives no information on the shear rate for this molecularly thick layer. Modified AFM techniques provide valuable information on confined liquids, but the major drawback is that mean contact pressure is in GPa range.

To better characterize rheological mechanisms during shearing in confined contacts, an important amount of instrumental development arises from the coupling of shearing experiments with in situ probes: FTIR, scattering devices, optical imaging, or spectroscopy. These couplings help to quantify molecular orientation and relaxation in a shear field $^{27,28}$ or to detect in real time molecular reorientation in confined liquids. ${ }^{29}$ Spectroscopic methods with fluorescent dyes in a confined film were also developed to study the molecular relaxation after normal or lateral stress. ${ }^{30,31} \mathrm{It}$ was measured on liquids by Frantz et al. but the maximum normal load is rather small $(50 \mu \mathrm{N}) .^{30}$ Still using this coupling, Mukhopadhyay et al. tried to correlate diffusion coefficient of the fluorescent molecules and molecular friction in the confined liquid. ${ }^{31}$ Fluorescence techniques are good candidates to estimate molecular diffusion coefficient and/or velocity down to molecular scale. Lateral motion (molecular diffusion or molecular local velocity) of fluids in relative motion is usually studied with fluorescence recovery after photobleaching (FRAP) methods..$^{32,33}$ This technique was coupled with SFA to investigate the diffusion coefficient of polymer chains under confinement but no shear motion was added. ${ }^{34}$ Meanwhile, this technique allows to estimate shear flow depending on the surface-liquid interaction. It can be extended to LB bilayers studies in order to estimate a local molecular friction. ${ }^{35}$ Moreover, the introduction of an intensity separation interferometer on the FRAP device, which provides an interference fringe pattern (technique FRAPP_Fluorescence Recovery After Patterned Photobleaching), allows to investigate diffusion phenomena at well defined length scales and leads to a more precise characterization of local motion. ${ }^{36-40}$

All in all, this evidenced a lack of the analysis of friction of highly confined films for elastic mean contact pressure above $10 \mathrm{MPa}$ over micrometer scale contact. To get low pressure range, high contact areas were reached by Sfarghiu et al. who derived a macro-tribometer with fluorescence probes on phospholipid bilayers. ${ }^{41}$ Their measurements are made in a liquid cell and the analysis gives an average information on shearing. Experimental apparatus which can provide shear rate information and local shear stress versus mean contact pressure in the meantime are scarce. This was the starting point of our study. This study will present the interest of coupling two setups: a nano-sclerometer to get in situ shearing information and a velocimetry device based on fluorescence photobleaching (FRAPP) which give information on the velocity field. The major challenge is to get velocity information on a nanometer scale layer confined and sheared over a micrometer scale contact area.

\section{EXPERIMENTAL}

To investigate shearing of confined ultra-thin films at a controlled mean contact pressure, a novel coupling of two setups was performed: a nano-sclerometer with in situ observation of the contact which monitors the confinement coupled with a FRAPP setup which is usually used to record molecular diffusion and velocity flow. ${ }^{36,37,39}$ The final aim is to estimate both the strain rate and the shear rate of the confined layer ensuring in situ observation. The nano-sclerometer, based on a custom sclerometer setup whose basic measuring principle is fully described elsewhere, ${ }^{42}$ is custom-made. Control of the sliding motion and recording of the normal load $F_{n}$, tangential force $F_{t}$, and speed $v$ are computer driven. Experimental measurements give the friction coefficient $\mu$ which equals $F_{t} / F_{n}$. A built-in microscope allows in situ control and analysis of the contact area between the tip and the surface. From these in situ observations, we can deduce a mean value of the contact area $A$, the mean contact pressure $p_{\text {mean }}=F_{n} / A$, and the shear stress $\tau=F_{t} / A=\mu p_{\text {mean }}$. Figure 1(a) shows a schematic illustration of the coupling system. We adapted our sclerometer device with a NTR2 nano-tribometer (AntonPaar Tritec) for which both normal load and tangential force ranges go from $0.5 \mu \mathrm{N}$ up to $1 \mathrm{~N}$ (normal force resolution: $0.1 \mu \mathrm{N}$, tangential force resolution: $1 \mu \mathrm{N})$. The spherical indenters were 51 or $25 \mathrm{~mm}$ in radii and consisted of borosilicate BK7 precision lenses polished to tight tolerances (Newport $($ ). Lateral motion is controlled by a linear table (Schneeberger®, $\left.1 \mu \mathrm{m} / \mathrm{s} \leq V_{i} \leq 10 \mathrm{~mm} / \mathrm{s}\right)$. There is no climatic chamber in this first approach. The FRAPP setup is similar to the one described by Davoust et ll $^{36}$ based on amplitude splitting interferometer. An argon laser (Spectra Physics, $\lambda=488 \mathrm{~nm}$ ) is divided into two beams by a semi-reflective blade. One beam goes directly to the sample, while the other is reflected on a piezoelectrically modulated mirror and redirected to the sample. The two crossing beams are coherent and an interference pattern is created on the sample. For the coupling, the main issue was to have both in situ observation of the contact and recording of the fluorescence signals. We choose to separate the optical path of the sclerometer camera from the laser beams. Figure 1(b) displays the contact area of a $25 \mathrm{~mm}$ radius indenter on a glass slide $\left(F_{n}=1 \mathrm{~N}\right)$ and the fringe pattern at the surface (here fringe spacing is $\sim 90 \mu \mathrm{m})$. As on a classical FRAPP experiment, the fringe spacing $i$ and the related wave vector $q=2 \pi / i$ are related to the crossing angle $\theta$ between the two beams by $i=\lambda / 2 \sin (\theta / 2)$. By adjusting the distance $a$ between the mirror and the semi-reflective blade, one can easily change the 

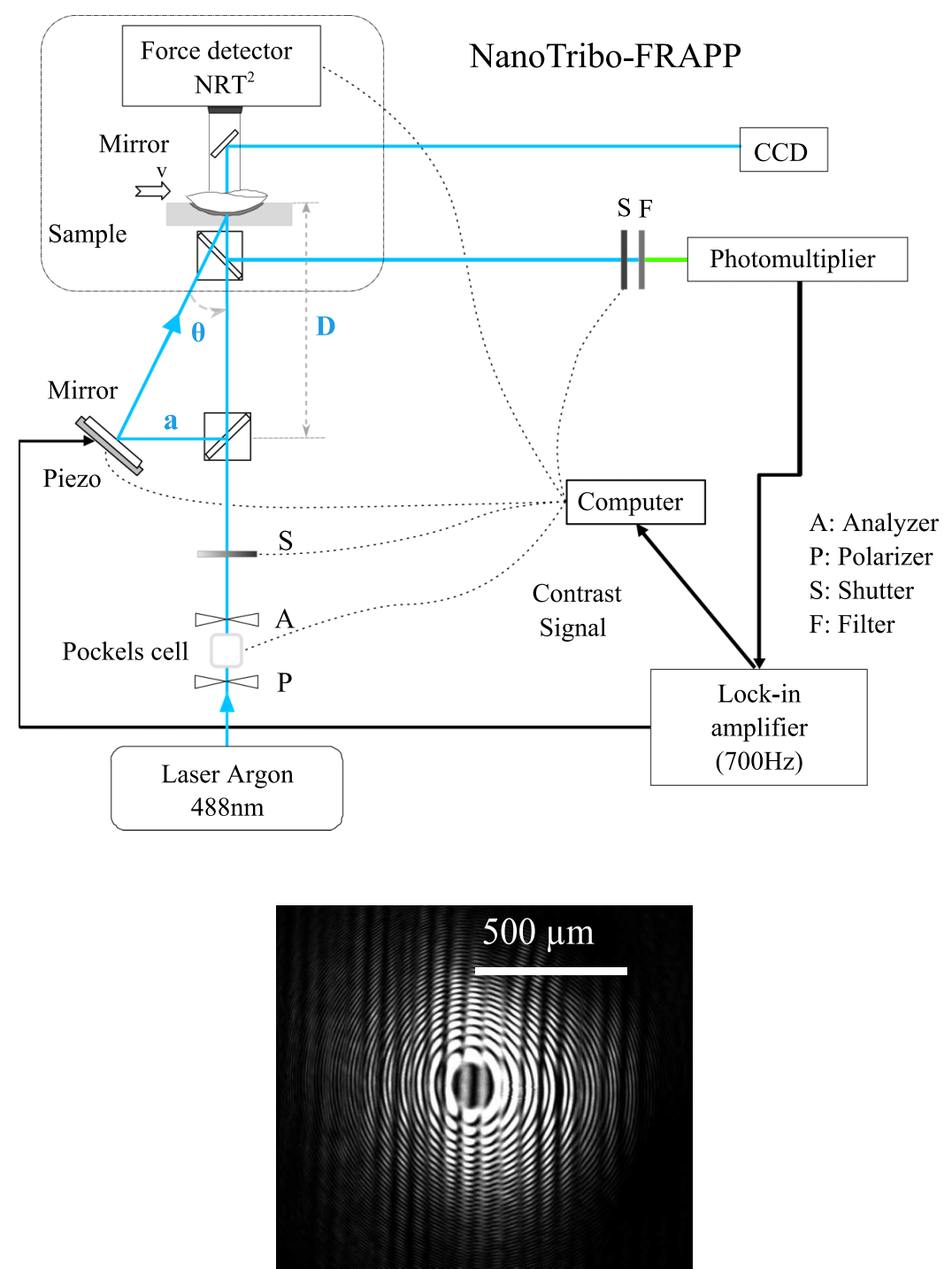

FIG. 1. (a) Schematics of the NanoTribo-FRAPP setup; (b) contact area of a $25 \mathrm{~mm}$ radius indenter on a glass slide and fringe pattern.

angle $\theta$ and control fringe spacing $i$ from $8 \mu \mathrm{m}$ to $100 \mu \mathrm{m}$ in our case.

The illuminated area is approximately $1.5 \mathrm{~mm}$ square which is sufficient enough for contact diameter of hundreds of micrometers. One critical point is to get enough fluorescence signal from the contact area sheared compared to the overall bleached area, and that is why we add a rectangular mask under the sample to increase the signal to noise ratio. The recorded signals are the tangential force versus time for the nano-tribo setup. Knowing the contact area $A$, this signal allows to calculate the shear stress $\tau$ at the interface. On the FRAPP setup, the contrast of the fringe pattern is detected by modulation of the illuminating fringes position at $700 \mathrm{~Hz}$, by means of a sinusoidal tension excitation of the piezoelectrical crystal. A lock-in amplifier is used to filter the received signal by selecting the characteristic frequency of the piezo-electrical vibration. The experiment occurs in two steps. First, the fringe pattern is printed on the studied substrate: fluorescence photobleaching of the labeled species in the illuminated fringes is created by hint of a Pockels cell which produces a full intensity pulse on the sample. Second, the laser intensity returns to a low level and simultaneously both shearing and mirror's oscillation start, and the emerging fluorescence signal is then collected by a photomultiplier.

Following the work of Davoust et al. ${ }^{36}$ the fluorescence signal $F(t)$ is related to the fluorescent probe concentration $c(\mathbf{r}, t)$ and to the reading intensity $I(\mathbf{r}, t)$ by

$$
F(t)=\int c(\mathbf{r}, t) I(\mathbf{r}, t) \mathrm{d}^{3} \mathbf{r}=\int \tilde{c}(\mathbf{q}, t) \tilde{I}(-\mathbf{q}, t) \mathrm{d}^{3} \mathbf{q},
$$

where $\tilde{c}(\mathbf{q}, t)$ and $\tilde{I}(\mathbf{q}, t)$ are the spatial Fourier transforms of $c(\mathbf{r}, t)$ and $I(\mathbf{r}, t) . F(t)$ is related to the mean contrast between dark and bright fringes. As the signal is non-monochromatic, $F(t)$ can be decomposed into a harmonic series in regard to the fundamental modulating frequency; in this experiment, the odd and even harmonic components, respectively, $f_{1}(t)$ and $f_{2}(t)$ are accessible simultaneously (Fig. 2(a), an example of a recorded $f_{2}(t)$ ). First, a Brownian diffusion behavior will be characterized by an exponential decay with a characteristic time $\tau_{q}=1 / D q^{2}$ where $D$ is the diffusion coefficient. Second, a drift velocity $v_{d}$ of the fluorescent probes will give an oscillation of the signal with a frequency $f=i / v_{d}{ }^{36}$ where $i$ is the imposed fringe spacing. Experimentally, we can access this typical frequency by simply fitting the data. A more refined fast 

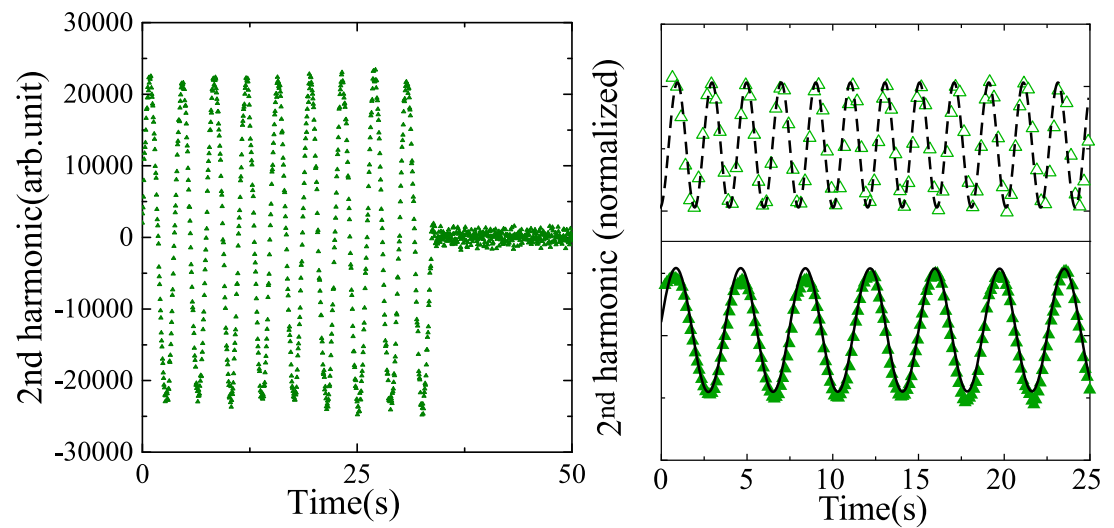

FIG. 2. (a) Contrast signal versus time curve recorded by the FRAPP setup where the epoxy sample moves at a given speed (for this example: $10 \mu \mathrm{m} / \mathrm{s}$ during $\sim 30 \mathrm{~s}$ ); (b) fitting of the recorded contrast signal for two fringe spacings for the epoxy sample ( $i=20 \pm 2 \mu \mathrm{m}$ and $38 \pm 2 \mu \mathrm{m}$, respectively).

Fourier transform analysis can be used to highlight a (or a series of) characteristic frequency related to the velocity profile as previously described by Davoust et $a l .{ }^{36}$ When comparing the FRAPP curves obtained with and without shear, one can determine the flow velocity under shear by $v_{\text {mes }}=f \times i$.

\section{RESULTS AND DISCUSSION}

\section{A. Experimental validation of the setup: No contact, no shear stress applied}

To validate our setup, we conducted a first test without contact. A glass slide was covered by an epoxy film (Araldite 2020, $1 \mathrm{~mm}$ thick) homogeneously labelled with fluorescein sodium salt (Aldrich chemical). Epoxy was chosen because of his stable physical and chemical properties. Meanwhile, the fluorescent dyes incorporated present almost no diffusion, which facilitate our measurement of flow velocity. The sample was fixed directly on the force detector NTR2. Then the system was set to move and the contrast signal was recorded. Here we tested 3 imposed speeds $\left(V_{i}=1 \mu \mathrm{m} / \mathrm{s}, 10 \mu \mathrm{m} / \mathrm{s}\right.$, and $100 \mu \mathrm{m} / \mathrm{s}$ ) for 2 interfacial fringe spacings $(i=20 \pm 2 \mu \mathrm{m}$ and $38 \pm 2 \mu \mathrm{m}$ ). Figure 2(a) displays the recording of the second harmonic $f_{2}$ versus time. The motion was stopped after $30 \mathrm{~s}$. First, when motion is stopped, the recorded contrast signal falls to zero clearly demonstrating that the recorded signal is related to the motion of the sclerometer device. Second, during motion, the periodic signal should be linked to the sliding speed $V_{\text {mes }}=f i$ where $f$ is the sinusoidal frequency and $i$ the fringe spacing. Knowing the fringe spacing, the curve is fitted with a damped sine wave. As seen in the curves of Fig. 2(b), for a given speed at $10 \mu \mathrm{m} / \mathrm{s}$, two fringe spacings

TABLE I. Fitting results of the recorded contrast signal for two different fringe spacings for the epoxy sample.

\begin{tabular}{lccc}
\hline \hline$V_{i}(\mu \mathrm{m} / \mathrm{s})$ & $i(\mu \mathrm{m})$ & $f(\mathrm{mHz})$ & $V_{\text {mes }}(\mu \mathrm{m} / \mathrm{s})$ \\
\hline 1 & $20 \pm 2$ & $49.5 \pm 0.5$ & $0.98 \pm 0.2$ \\
1 & $38 \pm 2$ & $26.7 \pm 0.1$ & $1 \pm 0.5$ \\
10 & $20 \pm 2$ & $490 \pm 2$ & $9.7 \pm 1$ \\
10 & $38 \pm 2$ & $266 \pm 1$ & $10 \pm 0.6$ \\
100 & $73 \pm 2$ & $1380 \pm 10$ & $98 \pm 3$ \\
100 & $111 \pm 2$ & $900 \pm 50$ & $100 \pm 2$ \\
\hline \hline
\end{tabular}

( $i=20 \pm 2 \mu \mathrm{m}$ and $38 \pm 2 \mu \mathrm{m}$, respectively) lead to two characteristic frequencies. Table I sums up the fitting results: the recorded signal matches the imposed sliding speed $V_{i}$. The fast Fourier transform of the signal for each tested sliding speed is calculated. From these calculated frequencies, the velocities spectra are extracted. Figure 3 displays normalized FFT intensity versus the measured velocity $V_{\text {mes }}$ for those two mentioned interfacial spacings. These results show that the measured velocity and the imposed sliding speed $V_{i}$ are in agreement, providing a good validation of our experimental setup.

In a second step, a same configuration was performed on a model phospholipid monolayer. We chose DSPC $(1,2-$ distearoyl-sn-glycero-3-phosphocholine, Avanti Polar Lipids) and $1 \%$ DSPC labeled with NBD-DPPE fluorophore $(1,2-$ dipalmitoyl-sn-glycero-3-phosphoethanolamine-N-(7-nitro-21,3-benzoxadiazole-4-yl, Avanti Polar Lipids). The transition

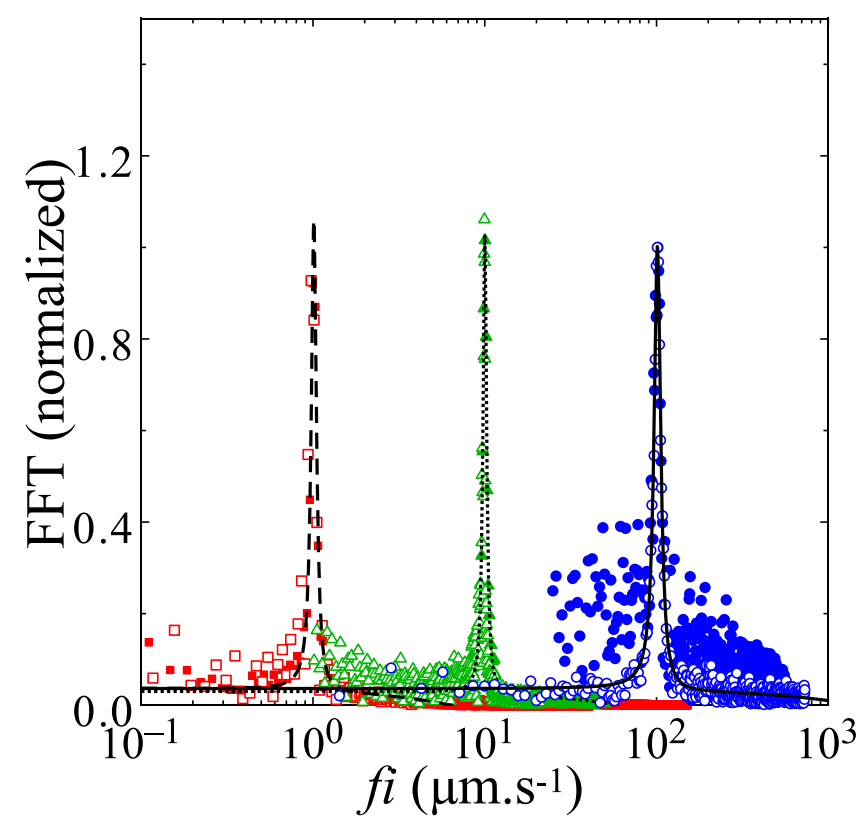

FIG. 3. Validation test: normalized FFT intensity versus measured velocity $V_{\text {mes }}=f i$ for the fluorescently labeled epoxy sample: (red open square) $V_{i}=1 \mu \mathrm{m} / \mathrm{s}, i=38 \mu \mathrm{m}$; (red solid square) $V_{i}=1 \mu \mathrm{m} / \mathrm{s}, i=20 \mu \mathrm{m}$; (green open triangle) $V_{i}=10 \mu \mathrm{m} / s, i=38 \mu \mathrm{m}$; (green solid triangle) $V_{i}=10 \mu \mathrm{m} / \mathrm{s}$, $i=20 \mu \mathrm{m}$; (blue open circle) $V_{i}=100 \mu \mathrm{m} / \mathrm{s}, i=111 \mu \mathrm{m}$; (blue solid circle) $V_{i}=100 \mu \mathrm{m} / \mathrm{s}, i=73 \mu \mathrm{m}$. 


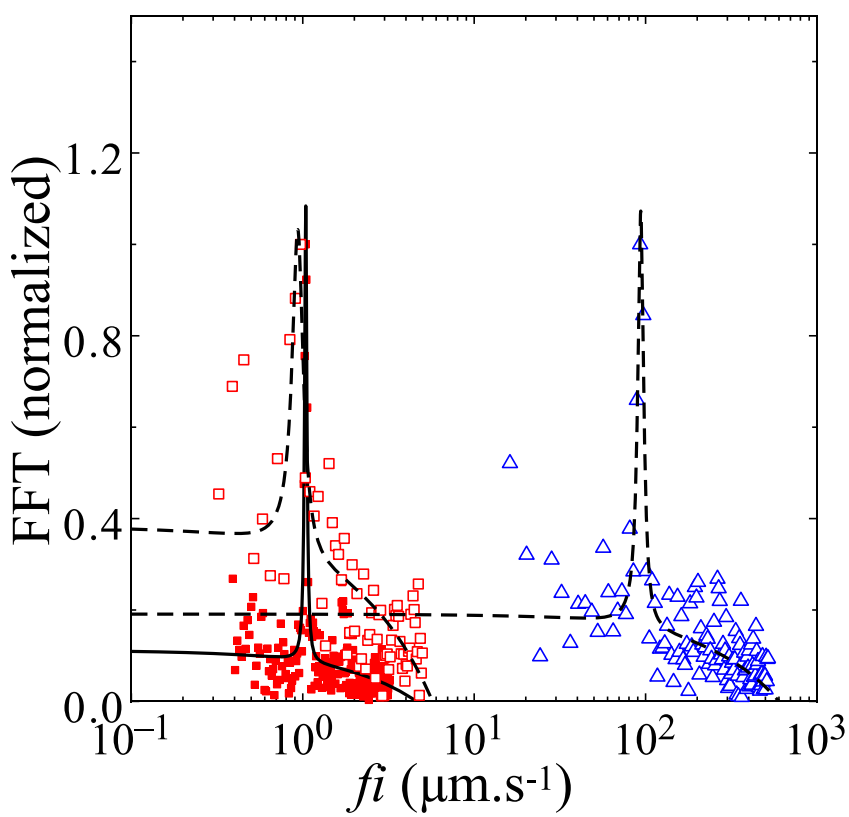

FIG. 4. Normalized FFT intensity versus measured velocity $V_{m e s}=f i$ for the supported fluorescently labeled DSPC monolayer: (red open square) $V_{i}=1 \mu \mathrm{m} / \mathrm{s}, i=106 \mu \mathrm{m}$; (red solid square) $V_{i}=1 \mu \mathrm{m} / \mathrm{s}, i=20 \mu \mathrm{m}$; (blue open triangle) $V_{i}=100 \mu \mathrm{m} / \mathrm{s}, i=106 \mu \mathrm{m}$.

temperature of the DSPC bilayer in water is $55^{\circ} \mathrm{C}$ and it acts as a solid-like layer of 3-4 nanometers at ambient temperature. This system has a low diffusion coefficient and then the flow velocity can easily be extracted from the harmonic curves $f_{1}(t)$ and $f_{2}(t)$. The monolayer was formed on a Langmuir-Blodgett trough and transferred on a clean microscope glass slide (sonicated successively in chloroform, acetone, ethanol, and Milli-Q water for 15 min each). Results shown in Fig. 4 reveal that the measured velocity also concurs with the imposed sliding speed $V_{i}$ and are summarized in Table II. The peak bases for the monolayer are clearly wider than those for the epoxy, and this is more evident for a higher imposed speed. The fact is due to the FFT which is calculated over a finite range. Experimentally the maximum length to get information during shearing depends on the bleached area
TABLE II. Fitting results of the recorded contrast signal for two fringe spacings for the DSPC monolayer sample.

\begin{tabular}{lrcc}
\hline \hline$V_{i}(\mu \mathrm{m} / \mathrm{s})$ & $i(\mu \mathrm{m})$ & $f(\mathrm{mHz})$ & $V_{\text {mes }}(\mu \mathrm{m} / \mathrm{s})$ \\
\hline 1 & $20 \pm 2$ & $53.5 \pm 1$ & $0.99 \pm 0.1$ \\
1 & $106 \pm 2$ & $9.4 \pm 2$ & $1 \pm 0.3$ \\
100 & $106 \pm 2$ & $943 \pm 26$ & $96 \pm 4$ \\
\hline \hline
\end{tabular}

which is approximately $1.5 \mathrm{~mm}$ length. Higher speed, shorter time range can be analyzed. It is therefore not possible for the moment to have a more accurate signal for each given system. Meanwhile, Figure 4 shows more expanded peak bases for tests on the DSPC monolayer. This is a result of a lower content of fluorescein in the monolayer system, leading a lower signalto-noise ratio. This can be slightly improved by adjusting the photomultiplier setups.

\section{B. Diffusion coefficient and velocity profile of a polymer solution: No contact, shear stress applied}

To test our configuration in a more complex situation, we investigate the rheological properties of a solution of linear, water-soluble non-ionizable polymer (Poly(Ethylene Glycol) PEG, molecular weight $20000 \mathrm{~g} \mathrm{~mol}^{-1}$ from Merk) with a concentration of $1 \%$ wt. A small fraction of the PEG is fluorescently labeled by Fluorescein (Fluorescein (methyl-PEGFITC) from Nanocs, Inc., molecular weight $20000 \mathrm{~g} \mathrm{~mol}^{-1}$ ). A drop of solution is put between a glass slide and a spherical indenter of radius $R=51 \mathrm{~mm}$ separated by a distance $h=1 \mathrm{~mm}$, in near plane Couette flow geometry $(h \gg R)$. We first characterize the diffusion law without any shear and determine the diffusion coefficient of the polymer $D$ $=(4.8 \pm 0.3) \cdot 10^{-11} \mathrm{~m}^{2} \mathrm{~s}^{-1}$ (see Figure 5(a)).

In a second step, we apply a velocity $v_{0}$ to the tip and try to characterize the velocity profile of the polymer solution. To calculate the experimental signal $F(t)$ (Equation (1)), we need to know the evolution of the concentration in fluorescent probe, which is solution of the diffusion equation with a convection flow $v(z)$ and a diffusion coefficient $D$,

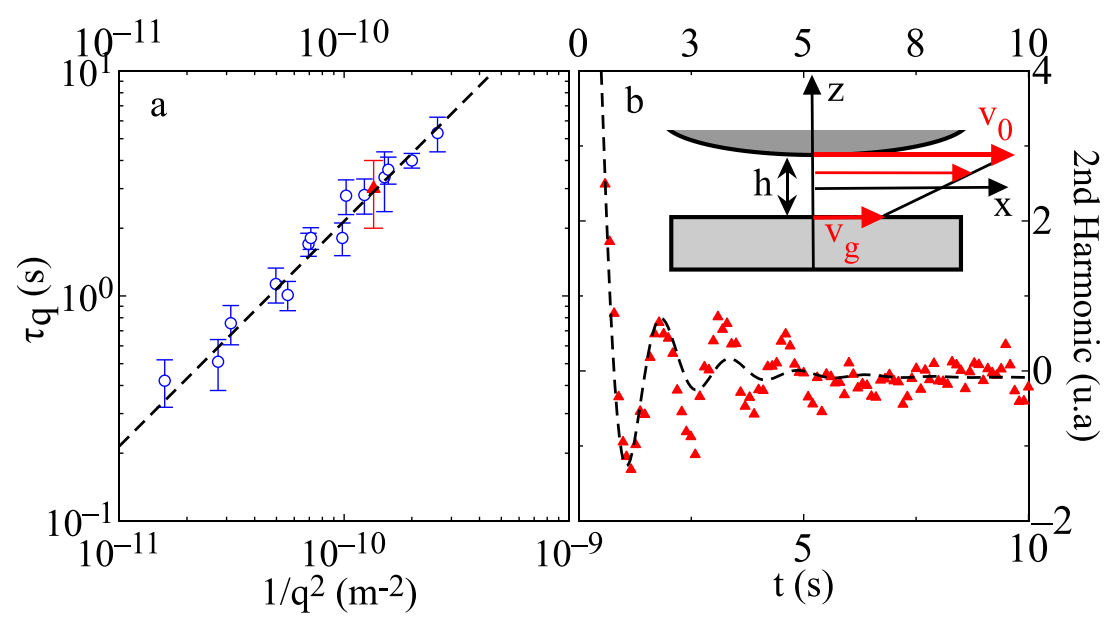

FIG. 5. (a) Diffusion characteristic time $\tau_{q}$ versus wave vector transfer for the studied polymer solution: (blue open circle) without any shear; (red solid triangle) with shear; (b) contrast signal and fit curve versus time curve recorded by the FRAPP setup where the solid tip is sliding the polymer solution at a given speed (50 $\mu \mathrm{m} / \mathrm{s})$. 


$$
\partial_{t} c(x, z, t)=D \Delta c(x, z, t)-v(z) \partial_{x} c(x, z, t),
$$

with a linear velocity profile,

$$
v(z)=\frac{v_{t}+v_{s}}{2}+\frac{v_{t}-v_{s}}{2 h} z .
$$

The boundary conditions are the velocity at the tip interface $v_{t}$ and the sliding velocity $v_{s}$ at the bottom surface. We also have to ensure the no-penetration of the fluorescent molecules at the boundaries. This last condition is difficult to ensure with an analytical solution for $c(x, z, t)$. We thus choose to approximate the concentration profile by the following expression:

$$
\begin{aligned}
c(x, z, t)= & c_{0} \exp \left\{-D q^{2} t\left(1+\frac{1}{3}\left(\frac{v_{t}-v_{s}}{2 h} t\right)^{2}\right)\right\} \\
& \times \cos \{q(x-v(z) t)\},
\end{aligned}
$$

corresponding to penetrating boundary conditions. After convolution of this concentration profile with the reading intensity $I_{0}(1+\cos \{q x+\Phi(t)\})(H(z+h / 2)-H(z-h / 2))$, where $H(z)$ is the Heaviside function, we obtain for the total fluorescence intensity,

$$
\begin{aligned}
I(t) \propto & \exp \left\{-D q^{2} t\left(1+\frac{1}{3}\left(\frac{v_{t}-v_{s}}{2 h} t\right)^{2}\right)\right\} \\
& \times \operatorname{sinc}\left\{q \frac{v_{t}-v_{s}}{4} t\right\} \cos \left\{q \frac{v_{t}+v_{s}}{2} t\right\} .
\end{aligned}
$$

The term in $t^{3}$ is characteristic of the coupling between the diffusion in $z$ direction and the convection flow.
Figure 5(b) shows the best fit obtained for a tip velocity $v_{0}=50 \mu \mathrm{m} \mathrm{s}^{-1}$ and fringe spacing $i=73 \mu \mathrm{m}$. We obtain $v_{t}$ $=49 \pm 2 \mu \mathrm{m} \mathrm{s}^{-1}, v_{s}=2 \pm 2 \mu \mathrm{m} \mathrm{s}^{-1}$, and $\tau_{q}=1 / D q^{2}=3 \pm 1 \mathrm{~s}$. It is in good agreement with non-sliding boundary conditions for the polymer flow. The characteristic time $\tau_{q}$ is also in very good agreement with the diffusion coefficient determined without any shear. Finally, it was impossible to have good fits with a plug flow and without taking into account the diffusion in $z$ direction. In this no contact configuration, assuming a linear profile and non-permeable boundary conditions, we were able to characterize a velocity profile together with a diffusion coefficient for a polymer solution, clearly demonstrating the capability of our experimental setup.

\section{Confinement and sliding experiment of a phospholipid monolayer: Contact, shear stress applied}

In order to prove the interest of our novel experimental coupling, we present some preliminary sliding friction results on a phospholipid monolayer. Langmuir had indicated that such a monolayer of about $3 \mathrm{~nm}$ of thickness is sufficient to reduce the shear stress of a smooth contact of the glass by a decade $^{43}$ (shear stress $\tau \sim 20 \mathrm{MPa}$ for a smooth contact of the glass under a mean contact pressure $p_{\text {mean }} \sim 30 \mathrm{MPa}$ ). Meanwhile, lipid layers also play an important role in the boundary lubrication mechanism of biological processes. Again,
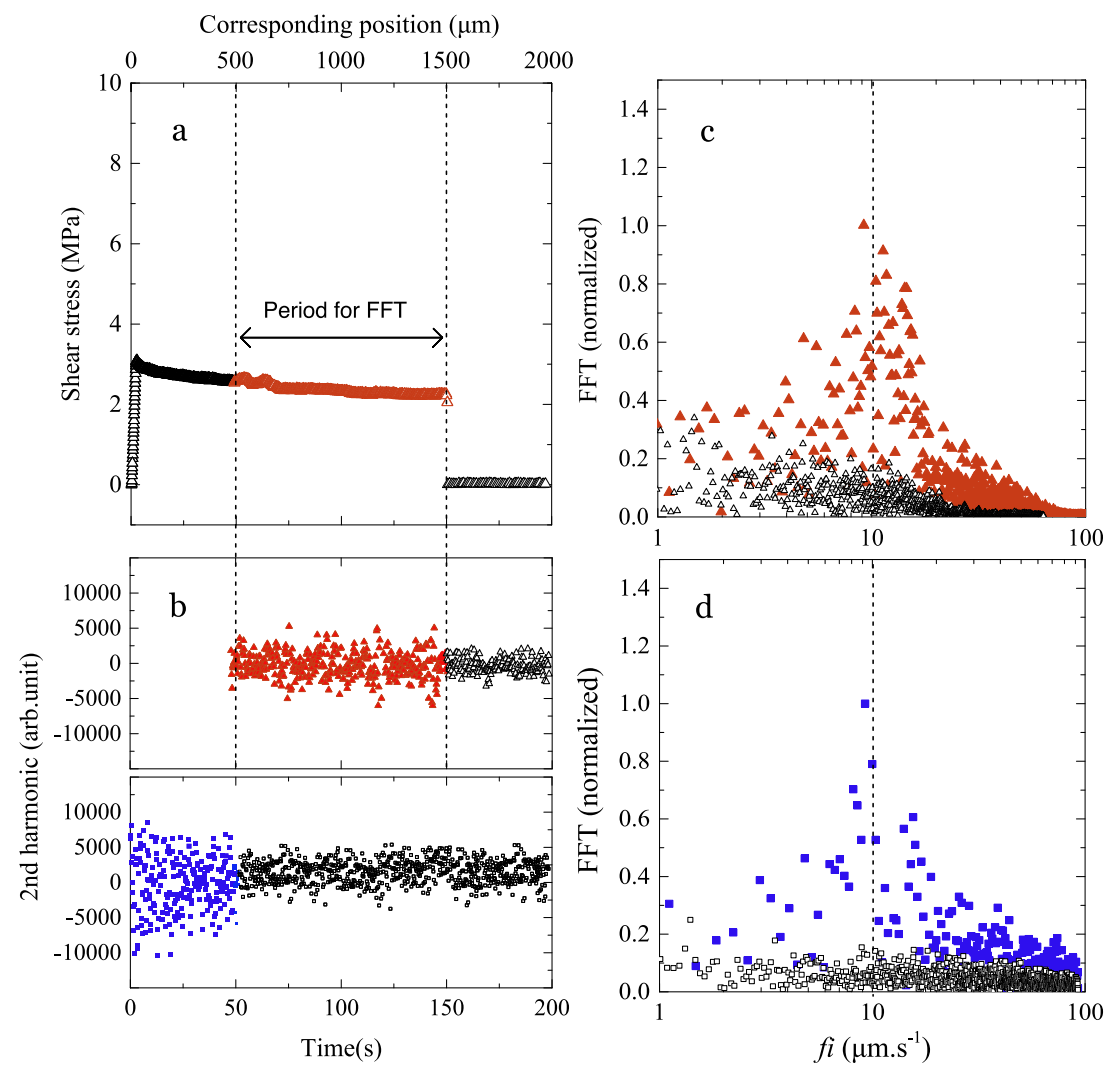

FIG. 6. Experiment on a sheared DSPC monolayer (triangles) for a contact pressure of $30 \pm 2$ MPa and comparison with the validation experiment without contact (squares, see Section III A) both at $v=10 \mu \mathrm{m} / \mathrm{s}$ and at ambient temperature: (a) shear stress against position; (b) contrast signals for a fringe spacing $=18.5 \pm 2 \mu \mathrm{m}$, (red solid triangle) with contact and shear (motion is stopped after $150 \mathrm{~s}$ ) and (blue solid square) validation experiment (motion is stopped after $50 \mathrm{~s}$ ); (c) (red solid triangle) corresponding normalized FFT intensity for the sheared DSPC monolayer, (open triangle) reference FFT analysis without any displacement after $150 \mathrm{~s}$; (d) (blue solid square) corresponding normalized FFT intensity for the validation experiment without contact, (open square) reference FFT analysis without any displacement after $50 \mathrm{~s}$. 
because of its relatively simple and stable structure and an easy achievement of a highly confined condition, the DSPC monolayer labeled with NBD fluorophore was chosen in this trial.

The contact was established between an indenter with a radius of $25 \mathrm{~mm}$ (Borosilicate BK7) and a glass slide supported DSPC monolayer as described above. The confinement is then controlled by the imposed normal load $F_{n}$ and the motion of the indenter is monitored. The tangential force $F_{t}$ and the normal contact area $A$ are recorded and then, the mean contact pressure $p_{\text {mean }}$ can be estimated. In this case, the imposed normal load is $1000 \mathrm{mN}$. From in situ observation of the contact, the mean pressure is $\sim 32 \mathrm{MPa}$. The experiments were performed at $20^{\circ} \mathrm{C}$ with a mean relative humidity around $50 \%$. The fringe spacing was set to $18.5 \mu \mathrm{m}$; meanwhile, we imposed a constant velocity of the moving tip at $10 \mu \mathrm{m} / \mathrm{s}$. On the shear stress versus position curve, a brief transient response followed by a stationary regime was shown in Figure 6(a), which is consistent with classical sliding tests. Usually stationary regime is deemed to occur after a few contact radius. In our instance, the contact radius is about $100 \mu \mathrm{m}$, and the stationary plateau is reached at $\sim 500 \mu \mathrm{m}$, which agree with each other. This information entails that the contrast signal from the FRAPP experiment will be integrated into the range [500-1500 $\mu \mathrm{m}$ ] (Figure 6(b)) to get, from the FFT, the velocity spectra shown in Figure 6(c). The velocity results show a Gaussian-like behavior which is consistent with the calculation limit discussed earlier: the finite range of the time domain induces noise during the conversion in the frequency domain. On the other hand, the spectra demonstrate a pronounced peak at about $10 \mu \mathrm{m} / \mathrm{s}$, which indicates that the majority of DSPC molecules under the tip are forced to move at this speed. At the same time, a corresponding validation test was conducted with a DSPC monolayer as described in Section III A, using a fringe spacing of $18.5 \pm 2 \mu \mathrm{m}$ and an imposed velocity of $10 \mu \mathrm{m} / \mathrm{s}$. The movement was kept for $50 \mathrm{~s}$ and the FFT result is shown in Figure 6(d). We observe a similar pronounce peak centered at the imposed velocity for both Figures 6(c) and 6(d). This may reveal that the lipids under the contact zone are moving at the imposed velocity.

\section{CONCLUSION}

We have developed an original experimental setup, coupling tribology (maximum normal load $1 \mathrm{~N}$ ) and velocimetry experiments (ranging from $1 \mu \mathrm{m} / \mathrm{s}-100 \mu \mathrm{m} / \mathrm{s}$ ) together with a direct visualization of the contact area. We present first test experiments, clearly demonstrating the interest of fast Fourier transform to analyze the fluorescence signals, allowing to measure drift velocity in the interfacial zone. We then use our experimental setup to characterize the shearing of a single lipid monolayer on a glass slide. In such a sheared nanometer thick interfacial layer, we are able to measure the local velocity for a contact diameter $\sim 200-300 \mu \mathrm{m}(\sim 30 \mathrm{MPa})$. We believe that this setup will help to light on the hydrodynamic of sheared confined layer in lubrication problems, as, for example, in lubrication, biolubrication, or friction on solid polymers.

\section{ACKNOWLEDGMENTS}

Support of the Region Alsace (GRAINE 2011) and of Labex NIE 11-LABX-0058-NIE (Investissement d'Avenir program ANR-10- IDEX-0002-02) is gratefully acknowledged. We thank F. Thalmann for stimulating discussions.

${ }^{1}$ B. N. Persson, Sliding Friction: Physical Principles and Applications (Springer Science \& Business Media, 2000), Vol. 1.

${ }^{2}$ F. MacKintosh and C. Schmidt, Curr. Opin. Colloid Interface Sci. 4, 300 (1999).

${ }^{3}$ S. Granick, Science 253, 1374 (1991).

${ }^{4}$ A. Mukhopadhyay and S. Granick, Curr. Opin. Colloid Interface Sci. 6, 423 (2001).

${ }^{5}$ U. L. B. Bhushan and J. N. Israelachvili, Nature 374, 607 (1995).

${ }^{6}$ L. Léger, H. Hervet, and R. Pit, "Friction and flow with slip at fluid-solid interfaces," ACS Symp. Ser. 781, 154-167, (2000).

${ }^{7}$ J. M. Georges, S. Millot, J. L. Loubet, and A. Tonck, J. Chem. Phys. 98, 7345 (1993)

${ }^{8}$ B. Cross, A. Steinberger, C. Cottin-Bizonne, J.-P. Rieu, and E. Charlaix, Europhys. Lett. 73, 390 (2006).

${ }^{9}$ S. Leroy, A. Steinberger, C. Cottin-Bizonne, A.-M. Trunfio-Sfarghiu, and E. Charlaix, Soft Matter 5, 4997 (2009).

${ }^{10}$ N. Dan, Curr. Opin. Colloid Interface Sci. 1, 48 (1996).

${ }^{11}$ J. N. Israelachvili and S. J. Kott, J. Colloid Interface Sci. 129, 461 (1989).

${ }^{12}$ J. Klein, D. Perahia, and S. Warburg, Nature 352, 143 (1991).

${ }^{13}$ J. Israelachvili, Surf. Sci. Rep. 14, 109 (1992).

${ }^{14}$ D. Chan and R. Horn, J. Chem. Phys. 83, 5311 (1985).

${ }^{15}$ J. Klein and E. Kumacheva, J. Chem. Phys. 108, 6996 (1998).

16. Jan Alsten and S. Granick, Phys. Rev. Lett. 61, 2570 (1988).

${ }^{17}$ C. D. Dushkin and K. Kurihara, Rev. Sci. Instrum. 69, 2095 (1998).

${ }^{18}$ B. J. Briscoe and D. C. B. Evans, Proc. R. Soc. London, Ser. A 380, 389 (1982).

${ }^{19}$ H. B. Kutzner, P. F. Luckham, and J. Rennie, Faraday Discuss. 104, 9 (1996).

${ }^{20}$ H. Darowska, M. Adams, B. Briscoe, and P. Luckham, Tribol. Ser. 38, 203 (2000).

${ }^{21}$ S. A. Joyce and J. Houston, Rev. Sci. Instrum. 62, 710 (1991).

${ }^{22}$ K. Feldman, M. Fritz, G. Hähner, A. Marti, and N. D. Spencer, Tribol. Int. 31, 99 (1998).

${ }^{23} \mathrm{R}$. Buzio and U. Valbusa, in Modern Research and Educational Topics in Microscopy, Microscopy Series No. 3, edited by A. Méndez-Vilas and J. Díaz (Formatex Research Center, 2007), Vol. 2, pp. 491-499, available at http://www.formatex.org/microscopy3/pdf/pp491-499.pdf.

${ }^{24}$ B. Bhushan, Springer Handbook of Nanotechnology (Springer Science \& Business Media, 2010).

${ }^{25}$ D. Gourdon, N. A. Burnham, A. Kulik, E. Dupas, F. Oulevey, G. Gremaud, D. Stamou, M. Liley, Z. Dienes, H. Vogel et al., Tribol. Lett. 3, 317 (1997).

${ }^{26}$ N. S. Tambe and B. Bhushan, Nanotechnology 16, 2309 (2005).

${ }^{27}$ M. Lucas and E. Riedo, Rev. Sci. Instrum. 83, 061101 (2012).

${ }^{28}$ I. Soga, A. Dhinojwala, and S. Granick, Langmuir 14, 1156 (1998).

${ }^{29}$ C. Y. Park, H. D. Ou-Yang, and M. W. Kim, Rev. Sci. Instrum. 82, 094702 (2011).

${ }^{30}$ P. Frantz, F. Wolf, X.-d. Xiao, Y. Chen, S. Bosch, and M. Salmeron, Rev. Sci. Instrum. 68, 2499 (1997).

${ }^{31}$ A. Mukhopadhyay, J. Zhao, S. C. Bae, and S. Granick, Rev. Sci. Instrum. 74, 3067 (2003).

${ }^{32}$ R. Pit, H. Hervet, and L. Léger, Tribol. Lett. 7, 147 (1999).

${ }^{33}$ A. Ponjavic, M. Chennaoui, and J. S. S. Wong, Tribol. Lett. 50, 261 (2013).

${ }^{34}$ J. S. S. Wong, L. Hong, S. C. Bae, and S. Granick, J. Polym. Sci., Part B: Polym. Phys. 48, 2582 (2010).

${ }^{35}$ R. Merkel, E. Sackmann, and E. Evans, J. Phys. 50, 1535 (1989)

36. Davoust, P. F. Devaux, and L. Léger, EMBO J. 1, 1233 (1982).

${ }^{37}$ K. B. Migler, H. Hervet, and L. Léger, Phys. Rev. Lett. 70, 287 (1993).

${ }^{38}$ L. Léger, H. Hervet, G. Massey, and E. Durliat, J. Phys.: Condens. Matter 9, 7719 (1997).

${ }^{39}$ E. Durliat, H. Hervet, and L. Léger, Europhys. Lett. 38, 383 (1997).

${ }^{40}$ L. Jourdainne, S. Lecuyer, Y. Arntz, C. Picart, P. Schaaf, B. Senger, J. C. Voegel, P. Lavalle, and T. Charitat, Langmuir 24, 7842 (2008).

${ }^{41}$ M.-C. Corneci, F. Dekkiche, A.-M. Trunfio-Sfarghiu, M.-H. Meurisse, Y. Berthier, and J.-P. Rieu, Tribol. Int. 44, 1959 (2011).

${ }^{42}$ C. Gauthier and R. Schirrer, J. Mater. Sci. 35, 2121 (2000).

${ }^{43}$ I. Langmuir, Trans. Faraday Soc. 15, 62 (1920). 